ORIGINAL RESEARCH

\author{
J.K. Dillon \\ C.M. Glastonbury \\ F. Jabeen \\ B.L. Schmidt
}

\title{
Gauze Padding: A Simple Technique to Delineate Small Oral Cavity Tumors
}

BACKGROUND AND PURPOSE: Small oral cavity tumors are an imaging challenge. Intimate apposition of vestibular oral mucosa to the alveolar mucosa makes tumor assessment difficult. In CT imaging, the "puffed cheek" method has been used to separate surfaces, though this is not feasible with long MR imaging sequences. We implemented placement of $2 \times 2$ inch $(6.45 \mathrm{~cm})$ gauze into the oral vestibule before the MR imaging examination, to determine whether this might improve tumor visualization.

MATERIALS AND METHODS: MR imaging examinations of all T1 oral malignant tumors treated at University of California, San Francisco, by the Oral and Maxillofacial Department were reviewed by 2 neuroradiologists. Nine patients were included in the final analysis. Six patients were imaged by using a standard protocol. Three patients were imaged with gauze placement. The radiologists evaluated the MR images, assessing whether they could see the tumor and then fully delineate it and its thickness.

RESULTS: Fisher exact analysis was performed on questions 1, 2, and 4 with the following results: $P$ value $=.048$, Can you see the tumor? $P$ value $=.012$, Can you fully delineate? $P$ value of .012 , How confident are you? MR imaging examinations with gauze clearly delineated the tumor with the tumor thickness measurable. MR imaging examinations without gauze did not clearly show the tumor or its thickness. Confidence of interpretation of the findings was also increased when gauze was used.

CONCLUSIONS: A $2 \times 2$ inch $(6.45 \mathrm{~cm})$ rolled gauze in the oral vestibule significantly improved tumor localization and delineation at MR imaging. This technique is simple and provides superior preoperative imaging evaluation and treatment planning of small oral cavity tumors.

ABBREVIATIONS: FS = fat-saturated; NR1 = neuroradiologist $1 ; \mathrm{NR} 2=$ neuroradiologist $2 ; \mathrm{OSCC}=$ oral squamous cell carcinoma; $\mathrm{PT} 1=$ pathological stage $\mathrm{T} 1$; TNM = tumor-node-metastasis

$\mathbf{0}$ SCC has a poor prognosis, despite significant advances in medicine and surgery during the past 50 years. ${ }^{1,2} \mathrm{~A}$ patient's overall prognosis is multifactorial. It depends on the location of the primary tumor, the tumor thickness, the depth of tumor invasion, histologic characteristics, and tumor stage. These factors are important predictors of cervical metastasis. ${ }^{3}$ Local recurrence of tumor in the oral cavity and regional lymph node metastasis halves the 5 -year survival rate ${ }^{4,5}$; however, the determinants of tumor recurrence and nodal metastasis are still not fully understood.

Small mucosal tumors of the oral cavity are a diagnostic challenge for both the head and neck surgeon and the head and neck radiologist. ${ }^{6}$ The oral cavity has a complex $3 \mathrm{D}$ anatomy, and even experienced neuroradiologists may find it difficult to first locate and then completely assess a small clinically $\mathrm{T} 1$ stage oral cavity primary tumor with confidence. Part of this difficulty is adequate visualization of the tumor due to the intimate apposition of the oral mucosa to the alveolar bone mucosa. Because these 2 anatomic boundaries are in contact, it

Received July 21, 2010; accepted after revision September 20

From the Departments of Oral and Maxillofacial Surgery (J.K.D.) and Radiology (F.J.), University of Washington, Seattle, Washington; and Departments of Radiology and Biomedical Imaging (C.M.G.), University of California, San Francisco, San Francisco, California; and Department of Oral and Maxillofacial Surgery (B.L.S.), New York University, New York, New York.

Previously presented as an oral abstract at: Annual Meeting of the American Society of Head and Neck Radiology, October 7-11, 2009; New Orleans, Louisiana.

Please address correspondence to Christine M. Glastonbury, MD, Department of Radiology, Otolaryngology-Head and Neck Surgery and Radiation Oncology, University of California, San Francisco, Box 0628, Room-358, 505 Parnassus Ave, San Francisco, CA 94143-0628; e-mail: christine.glastonbury@ucsf.edu

indicates article with supplemental on-line tables.

DOI 10.3174/ajnr.A2405

is difficult to see the subtle changes associated with a T1 tumor, adequately delineate the margins, and measure the thickness of the lesion. This can be even more of a challenge when the patient has multiple dental restorations or dental implants in place, adding to dental artifacts.

Imaging before surgery may be critical for the preoperative assessment and treatment planning of the patient. It can be used to determine the tumor thickness, depth of invasion, and possible bone infiltration and thus is used in preliminary tumor staging, which is regarded by some as the most important predictor of long-term patient prognosis. ${ }^{7}$

The "puffed cheek" method of separating oral cavity mucosal surfaces by distending the cheeks with air has been used successfully with CT imaging of oral cavity tumors; however, it is extremely difficult for patients to manage with long MR imaging sequences. ${ }^{6}$ At our institution, we prefer the added information that MR imaging provides over CT, specifically, better delineation of soft tissues and assessment of marrow infiltration and less obscuration of detail by dental artifacts. We sought to find a reasonable option to allow both the head and neck surgeon and the neuroradiologist to better localize and delineate the tumor. Gauze has a similar characteristic to air on MR imaging. It is low-signal-intensity on T1-weighted and T2-weighted images, unless saturated with saliva in which case it becomes hyperintense on T2-weighted images. We hypothesized that placing gauze into the oral cavity of patients with $\mathrm{T} 1$ stage OSCC before imaging would delineate T1 tumor involvement of the soft tissue and or adjacent alveolar bone and, therefore, be an adjunct to the assessment of $\mathrm{T} 1$ lesions by MR imaging. 

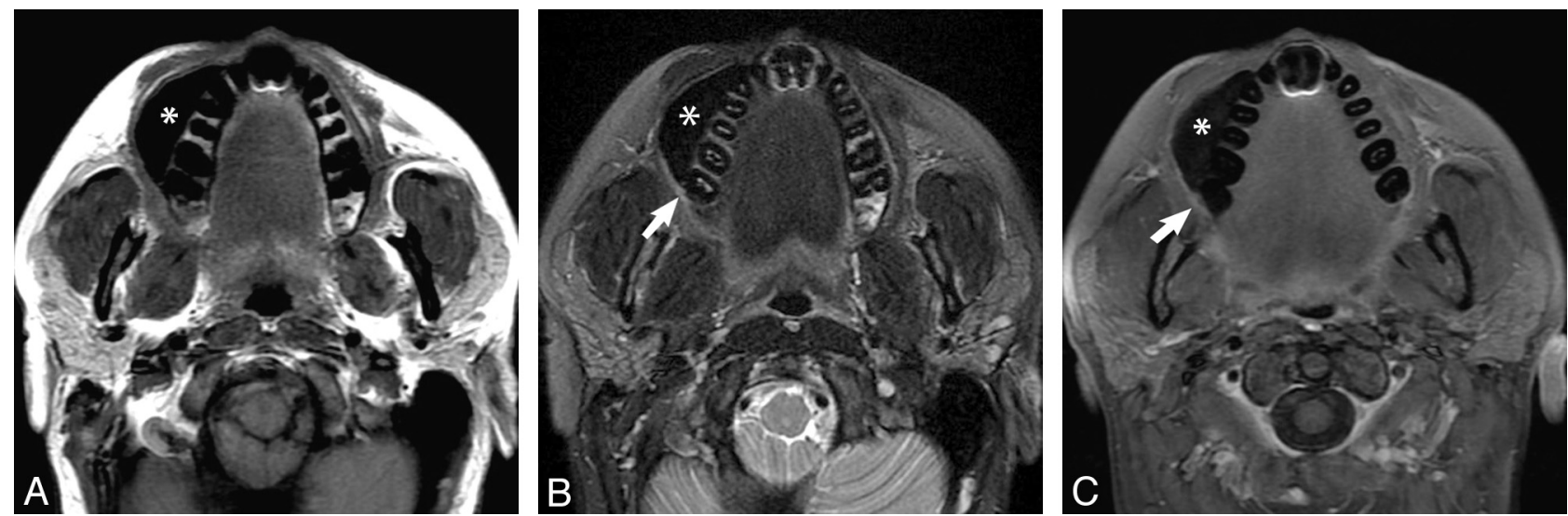

Fig 1. Noncontrast axial T1-weighted $(A)$, T2-weighted fat-saturated $(B)$, and postcontrast $T 1$ fat-saturated $(C)$ images demonstrate gauze padding (asterisk) distending the right oral vestibule and separating the 2 mucosal surfaces (ie, the buccal and the gingival surfaces, which would otherwise be apposed). The gauze is of low signal intensity on all sequences. A small primary OSCC is evident in the gingivobuccal sulcus (arrows in $B$ and $C$ ), and there is no evidence of deep infiltration. At surgery, this was proved to be a 2.1-mm-thick pT1 moderately differentiated OSCC
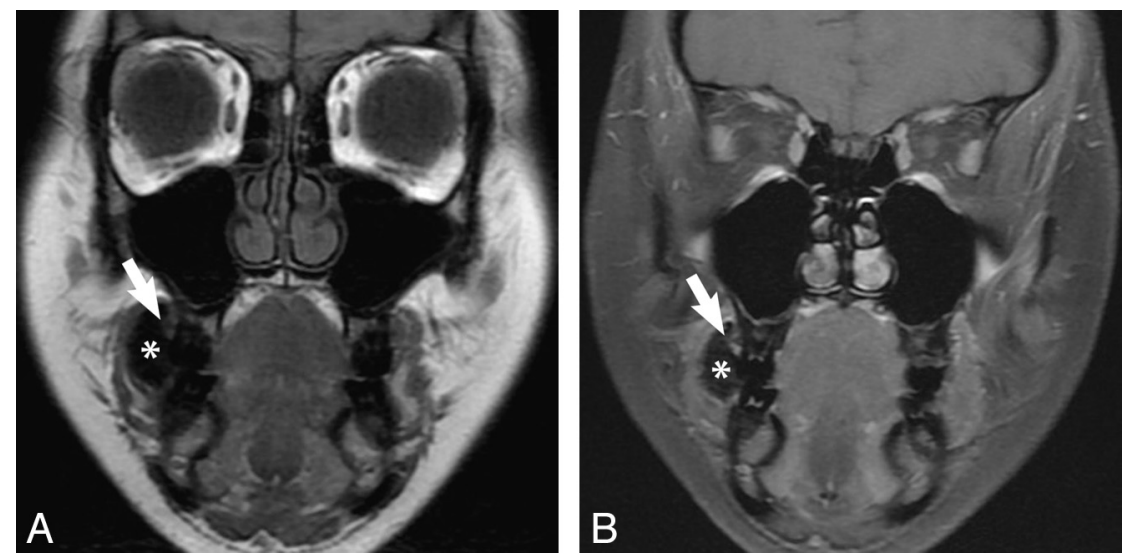

Fig 2. Coronal $\mathrm{T1}(A)$ and $\mathrm{T1}$ postcontrast FS MR $(B)$ images obtained with gauze in the right vestibule (asterisk) show subtle abnormal soft tissue, which enhances in the superior right gingivobuccal sulcus (arrow), illustrating a T1 buccal mucosal lesion. There is no evidence of extension medially into the maxilla or laterally into the buccinator muscle or cheek soft tissues Final pathology showed a well-differentiated OSCC pT1 and $3 \mathrm{~mm}$ thick.

\section{Materials and Methods}

All biopsy-proved clinical T1 buccal or gingival OSCCs treated by the Department of Oral and Maxillofacial Surgery at the University of California, San Francisco, between January 2004 and March 2009 in patients who underwent perioperative MR imaging were reviewed. One gauze procedure was performed in late 2003; 6 nongauze procedures, in 2006-2008; and the other 2 gauze procedures, in 2009. MR imaging was typically performed $2-4$ weeks after the biopsy, except in 1 patient in the nongauze group, when the scan was obtained $<1$ week after biopsy. The study was approved by the institutional review board.

Imaging for 8 patients was performed on a $1.5 \mathrm{~T}$ scanner (Signa EXCITE; GE Healthcare, Milwaukee, Wisconsin) by using a neurovascular head coil, and 1 of the nongauze procedures was performed on a 3T Signa EXCITE scanner. All scans were obtained with the following protocol: axial T1-weighted images with a section thickness of $5 \mathrm{~mm}$, section spacing of $1 \mathrm{~mm}$ by using an 18-cm FOV; and axial T2 FS images with a section thickness of $4 \mathrm{~mm}$, section spacing of 2 $\mathrm{mm}, 18$-cm FOV. Postcontrast T1-weighted FS images were acquired in the axial and coronal planes, both obtained at 5-mm section thickness with 1-mm spacing. Axial plane T1, T2 FS, and T1 + contrast and FS images were also obtained through the remainder of the neck. Despite a span of several years, the imaging parameters did not vary significantly among patients. No changes were made to the protocol for the gauze patients.

The patients who had imaging performed with the gauze in situ were shown how to roll up the $2 \times 2$ inch gauze square and place it into the oral vestibule adjacent to the $\mathrm{T} 1$ tumor. This procedure was demonstrated by the primary surgical team. Patients were then given a packet of gauze to take with them to the MR imaging scanner with instructions to place the gauze in their mouth just before commencement of the scanning. The MR imaging requisition form informed the neuroradiologist and technician of the gauze placement by the patient. Imaging that was degraded due to quality (ie, motion, artifacts, incomplete studies, and noncontrast examinations) was excluded from the study. This resulted in a total of 9 patients who could be included in the final analysis. Of the 9 patients reviewed, 6 had imaging performed without gauze (ie, the standard protocol in place for evaluation of $\mathrm{T} 1$ oral cavity lesions) and 3 had MR imaging with the gauze in place.

Two radiologists, 1 Certificate of Added Qualification-certified neuroradiologist and 1 neuroradiology fellow, blinded to the final staging, were asked to review the MR images independently on a PACS workstation. They were provided with the information that this was a buccal or gingival OSCC and were asked 4 simple questions:

Can you see the tumor? Can you fully delineate the tumor? For 


\begin{tabular}{|c|c|c|c|c|c|c|c|c|}
\hline \multirow[b]{2}{*}{ Gauze } & \multicolumn{2}{|c|}{$\begin{array}{l}\text { Can You } \\
\text { See It? }\end{array}$} & \multicolumn{2}{|c|}{$\begin{array}{c}\text { Can You } \\
\text { Delineate It? }\end{array}$} & \multicolumn{2}{|c|}{$\begin{array}{c}\text { How Confident } \\
\text { Are You? }\end{array}$} & \multicolumn{2}{|c|}{$\begin{array}{l}\text { How Thick } \\
\text { Is It? (mm) }\end{array}$} \\
\hline & NR1 & NR2 & NR1 & NR2 & NR1 & NR2 & NR1 & NR2 \\
\hline No & Yes & Yes & No & Yes & 1 & 1 & \multicolumn{2}{|c|}{ Cannot say } \\
\hline No & Yes & No & No & No & 0 & 0 & \multicolumn{2}{|c|}{ Cannot say } \\
\hline No & No & No & No & No & 0 & 0 & \multicolumn{2}{|c|}{ Cannot say } \\
\hline No & No & No & No & No & 0 & 0 & \multicolumn{2}{|c|}{ Cannot say } \\
\hline No & No & No & No & No & 0 & 0 & \multicolumn{2}{|c|}{ Cannot say } \\
\hline No & No & No & No & No & 0 & 0 & \multicolumn{2}{|c|}{ Cannot say } \\
\hline Yes & Yes & Yes & Yes & Yes & 3 & 3 & 5.0 & 5.5 \\
\hline Yes & Yes & Yes & Yes & Yes & 2 & 2 & 2.1 & 2.4 \\
\hline Yes & Yes & Yes & Yes & Yes & 3 & 3 & 4.1 & 5.6 \\
\hline$P$ value & \multicolumn{2}{|c|}{.048} & \multicolumn{2}{|c|}{.012} & \multicolumn{2}{|c|}{.012} & & \\
\hline
\end{tabular}

these 2 questions, they were asked for a yes/no response. How thick is the tumor? Finally, how confident are you with your findings? These results were recorded on an ordinal scale:

$0=$ Not confident/unable to see tumor

$1=$ Slightly confident/possibly able to see tumor

$2=$ More confident/probably able to see tumor

$3=$ Fully/very confident/fully able to see tumor.

A Fisher exact test was used to analyze the results.

\section{Results}

There were a total of 9 patients in the study (Table). Six patients did not have gauze placed. Three patients were not able to be imaged with gauze due to an inability to tolerate anything in the mouth due to severe pain from the lesion (1 patient) and gag reflex (2 patients). All of the procedures attempted with gauze were performed, and there were no increased motion artifacts on the images.

Three patients had gauze placed. In the no-gauze group, in only 2 cases did 1 or both of the neuroradiologists report being able to see the primary tumor. With the first case, both radiologists were able to see the tumor, but only NR2 could fully delineate it. When asked how confident they were in their findings, both were only slightly confident with their findings - the ordinal scale reported as 1 by both. In the second case, only NR1 reported seeing the tumor. Neither neuroradiologist could fully delineate it. They were also not confident in their findings - the ordinal scale reported as 0 by both. In the remaining 4 of the 6 cases of no gauze, both neuroradiologists were unable to see the tumor, were not able to fully delineate the tumor, and were not confident with their findings. Both neuroradiologists were also unable to measure the thickness of the tumor.

The neuroradiologists were able to see the tumor in all 3 cases with gauze and were able to fully delineate the tumor. In 2 of the 3 cases, they were fully/very confident of their findings- the ordinal scale reported as 3. In 1 case, they were more confident with their findings - the ordinal scale reported as 2. In these 3 cases, the neuroradiologists were also able to measure the tumor thickness, and they each had similar findings with an interobserver variability of $1-1.5 \mathrm{~mm}$.

With a Fisher exact test to compare the gauze and no gauze groups, the $P$ value was .048 for the first question, Can you see the tumor? The $P$ value was .048 for the questions, Can you fully delineate it, and How confident are you?

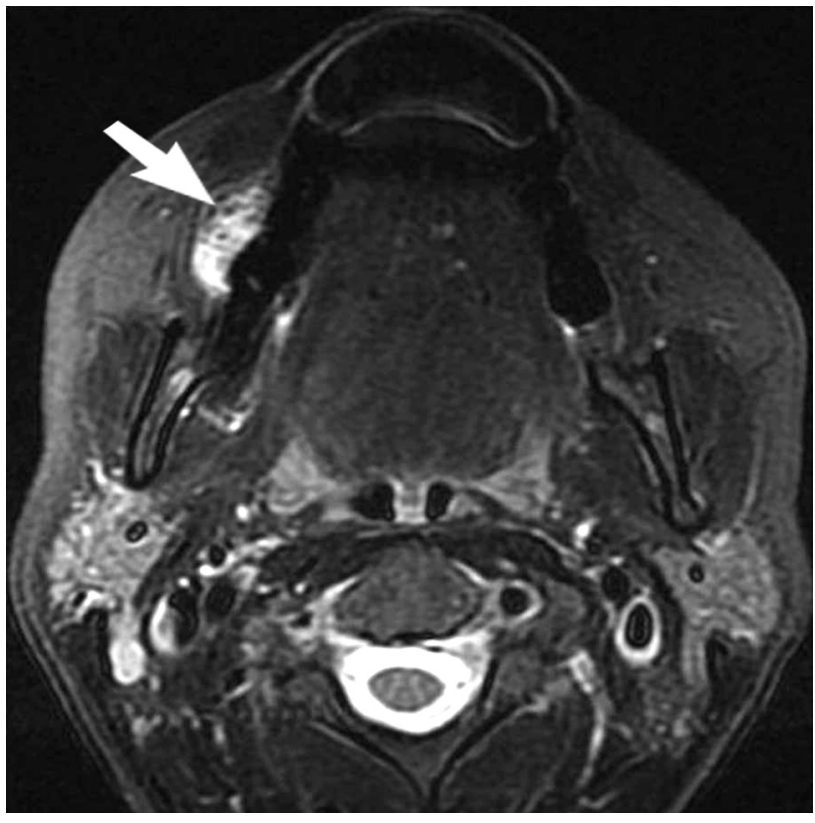

Fig 3. Axial T2-weighted fat-saturated image through the maxilla demonstrates high signal intensity within the gauze in the right oral vestibule (arrow). When the gauze is placed too early or if the patient has excessive salivation, it becomes soaked with saliva, resulting in hyperintense signal intensity. This does not interfere with distension of the vestibule or with evaluation of the postcontrast T1-weighted images.

\section{Discussion}

At least $90 \%$ of oral malignancy is OSCC. In the United States, it accounts for $2 \%-4 \%$ of the annually diagnosed malignancies with approximately 8000 deaths per year. ${ }^{8}$ Despite significant advances in research, surgical oncology, imaging, and postoperative critical care, the overall mortality is unchanged. ${ }^{8,9}$

The management of OSCC is still primarily surgical, ranging from resection with or without a neck dissection, followed by various combinations of radiation therapy or chemotherapy. Multiple factors have been studied regarding prognosis. ${ }^{2,10}$ Tumors $>2$ - to 4 -mm thickness, with close or positive surgical margins, depth of invasion $>2-4 \mathrm{~mm}$, and metastasis to locoregional lymph nodes have all been implicated as determinants of prognosis ${ }^{11}$; however, tumor thickness seems to be the only factor that has consistently shown promise as an indicator of risk for occult nodal metastasis. ${ }^{12-14}$ Tumors $>4$ $\mathrm{mm}$ have a greater likelihood of metastasizing to the neck. ${ }^{14}$

The status of the cervical lymph nodes is the single most important factor influencing patient survival. ${ }^{15,16}$ The TNM classification and staging, published by the American Joint Committee on Cancer, ${ }^{17}$ are an important part of overall assessment, treatment, and follow-up (On-Line Tables 1 and 2). OSCC has an overall 5-year survival rate of $48 \%$ for all stages. ${ }^{11}$ Lo et $\mathrm{al}^{18}$ reported the 5 -year survival rate for stages I, II, III, and IV disease to be $75 \%, 65.6 \%, 49 \%$, and $30 \%$ respectively. Sklenicka et al ${ }^{11}$ found a significant decrease in the survival for stage IV disease; however, their survival curves were not significantly different between stages I, II, and III. Full-thickness cortical invasion into the marrow by definition is stage IV disease. This invasion may not be easily appreciated on imaging if the overall lesion is small or if there are significant dental artifacts. The image findings, therefore, both have a prognostic implication and may also alter the surgical management. 
With modern advances in MR imaging, there has been significant improvement in the quality of images and sensitivity of findings. Section thickness can be as thin as $3 \mathrm{~mm}$. MR imaging is less affected by dental artifacts and has superior soft-tissue contrast compared with CT and is, therefore, more accurate in determining anatomic location and extension of OSCC. Techniques used to distend the oral vestibule, such as the puffed cheek ${ }^{19}$ and "water and contrast" techniques,"6 while suitable for CT scans, are not applicable to MR imaging due to the long imaging sequences.

This study shows that gauze, which has similar characteristics to air on MR imaging, can be successfully used to delineate T1 oral cavity tumor involvement of the soft tissue and/or adjacent alveolar bone (Figs 1 and 2). Most often, the person who has seen and biopsied the lesion initially is the surgeon. We found that it was simpler to instruct the patient on how to place the gauze adjacent to the tumor. This eliminated the need for the radiology technician to do this additional step, while also positioning the patient for the scan. Most important, the patient was instructed to place the gauze just before commencement of the scan so that the gauze would not become overly saturated with saliva, which would result in a T2 hyperintense appearance and possibly interfere with the final reading and reporting of the MR imaging (Fig 3).

The $2 \times 2$ inch size was chosen because it is large enough to distend the oral vestibule yet small enough to avoid soft-tissue distortion. While this is a small study, the puffed cheek technique of oral vestibule distension has been previously shown to be useful for CT imaging of oral cavity tumors. That technique is extremely difficult to use with MR imaging due to significantly longer scanning times. We have shown that cheek distension with gauze significantly improved the confidence of the radiologist when assessing oral cavity $\mathrm{T} 1$ lesions and provided a more detailed report, which allows more precise staging and treatment planning for the surgeon.

\section{Conclusions}

The placement of a $2 \times 2$ inch rolled gauze into the oral vestibule before imaging significantly improved identification of the lesion, tumor delineation, and confidence of interpretation of images; thus, it appears to be a useful technique for preoperative evaluation and treatment planning of oral cavity tumors. This method is inexpensive, requires no added imaging time, is typically painless for the patient, and requires minimal patient instruction.

\section{Acknowledgments}

We acknowledge Gary Sidhu, MD, for review of scans, Chirag Patel, MD, for figure support, Terry $\mathrm{Su}, \mathrm{MD}$, for data gathering, and Brian Christensen for table support.

\section{References}

1. Kademani D. Oral cancer. Mayo Clin Proc 2007;82:878-87

2. Sutton DN, Brown JS, Rogers SN, et al. The prognostic implications of the surgical margin in oral squamous cell carcinoma. Int J Oral Maxillofac Surg 2003;32:30-34

3. Patel RS, Clark JR, Dirven R, et al. Prognostic factors in the surgical treatment of patients with oral carcinoma. ANZ J Surg 2009;79:19-22

4. Batsakis JG. Surgical excision margins: a pathologist's perspective. Adv Anat Pathol 1999;6:140-48

5. Kademani D, Bell RB, Bagheri S, et al. Prognostic factors in intraoral squamous cell carcinoma: the influence of histologic grade. J Oral Maxillofac Surg 2005;63:1599-605

6. Fatterpekar GM, Delman BN, Shroff MM, et al. Distension technique to improve computed tomographic evaluation of oral cavity lesions. Arch Otolaryngol Head Neck Surg 2003;129:229-32

7. O'Brien CJ, Traynor SJ, McNeil E, et al. The use of clinical criteria alone in the management of the clinically negative neck among patients with squamous cell carcinoma of the oral cavity and oropharynx. Arch Otolaryngol Head Neck Surg 2000;126:360-65

8. Massano J, Regateiro FS, Januario G, et al. Oral squamous cell carcinoma: review of prognostic and predictive factors. Oral Surg Oral Med Oral Pathol Oral Radiol Endol 2006;102:67-76

9. Dillon JK, Liu SY, Patel CM, et al. Identifying risk factors for postoperative cardiovascular and respiratory complications after major oral cancer surgery. Head Neck 2011;33:112-16

10. Riley RD, Abrams KR, Sutton AJ, et al. Reporting of prognostic markers: current problems and development of guidelines for evidence-based practice in the future. Br J Cancer 2003;88:1191-98

11. Sklenicka S, Gardiner S, Dierks EJ, et al. Survival analysis and risk factors for recurrence in oral squamous cell carcinoma: does surgical salvage affect outcome? J Oral Maxillofac Surg 2010;68:1270-75. Epub 2010 Mar 29

12. Cheng A, Schmidt BL. Management of the N0 neck in oral squamous cell carcinoma. Oral Maxillofac Surg Clin North Am 2008;20:477-97

13. Simental AA Jr, Johnson JT, Myers EN. Cervical metastasis from squamous cell carcinoma of the maxillary alveolus and hard palate. Laryngoscope 2006;116:1682-84

14. Moore C, Kuhns JG, Greenberg RA. Thickness as prognostic aid in upper aerodigestive tract cancer. Arch Surg 1986;121:1410-14

15. Shah JP. Patterns of cervical lymph node metastasis from squamous carcinomas of the upper aerodigestive tract. Am J Surg 1990;160:405-09

16. Shah JP, Candela FC, Poddar AK. The patterns of cervical lymph node metastases from squamous carcinoma of the oral cavity. Cancer 1990;66:109-13

17. Edge SB, Compton CC. The American Joint Committee on Cancer: the 7th edition of the AJCC cancer staging manual and the future of TNM. Ann Surg Oncol 2010;17:1471-74

18. Lo WL, Kao SY, Chi LY, et al. Outcomes of oral squamous cell carcinoma in Taiwan after surgical therapy: factors affecting survival. J Oral Maxillofac Surg 2003;61:751-58

19. Weissman JL, Carrau RL. "Puffed-cheek" CT improves evaluation of the oral cavity. AJNR Am J Neuroradiol. 2001;22:741-44 